INPLASY

PROTOCOL

To cite: Xiaoli et al. Metaanalysis of fertility-preserving treatment in patients with early-stage endometrial cancer. Inplasy protocol 2020120137. doi:

10.37766/inplasy2020.12.0137

Received: 26 December 2020

Published: 27 December 2020

Corresponding author:

Zhao Sufen

252941051@qq.com

Author Affiliation:

Gynecology and Obstetrics, Department of the Second Hospital of Hebei Medical University, Shijiazhuang, China

Support: Self-raised funds.

Review Stage at time of this submission: Formal screening of search results against eligibility criteria.

Conflicts of interest:

None.

\section{Meta-analysis of fertility-preserving treatment in patients with early-stage endometrial cancer}

\author{
Zhao, XL1; Zhao, SF².
}

Review question / Objective: Meta-analysis of fertilitypreserving treatment in patients with early-stage endometrial cancer. The fertility-perserving treatment: a) taking oral progestin only therapy, b) hysteroscopic resection combined with progestin /LNG-IUS/GnRH-a, c) Levonorgestrel-releasing intrauterine system (LNG-IUS) or combined with progestin/ GnRH-a.

Condition being studied: The standard treatment for patients with early-stage EC is total hysterectomy and bilateral salpingo-oophorectomy with or without lymphadenectomy (according to the staging system of The International Federation of Gynecology and Obstetrics [FIGO] in 2009). Despite a higher 5-year survival rate (more than 90\%), it deprives the patients of their fertility permanently. Due to the late marriage and late childbearing, the universal two-child policy and other factors, an increasing number of young women are eager to retain reproductive function. Therefore, it is of vital importance to preserve reproductive function in the treatment of early-stage EC in young patients.

INPLASY registration number: This protocol was registered with the International Platform of Registered Systematic Review and Meta-Analysis Protocols (INPLASY) on 27 December 2020 and was last updated on 27 December 2020 (registration number INPLASY2020120137).

\section{INTRODUCTION}

Review question / Objective: Meta-analysis of fertility-preserving treatment in patients with early-stage endometrial cancer. The fertility-perserving treatment: a) taking oral progestin only therapy, b) hysteroscopic resection combined with progestin /LNGIUS/GnRH-a, c) Levonorgestrel-releasing intrauterine system (LNG-IUS) or combined with progestin/GnRH-a. 
Rationale: The purpose of this study was to compare the efficacy and safety of different fertility-preserving treatments for earlystage endometrial cancer.

Condition being studied: The standard treatment for patients with early-stage EC is total hysterectomy and bilateral salpingo-oophorectomy with or without lymphadenectomy (according to the staging system of The International Federation of Gynecology and Obstetrics [FIGO] in 2009). Despite a higher 5-year survival rate (more than $90 \%$ ), it deprives the patients of their fertility permanently. Due to the late marriage and late childbearing, the universal two-child policy and other factors, an increasing number of young women are eager to retain reproductive function. Therefore, it is of vital importance to preserve reproductive function in the treatment of early-stage EC in young patients.

\section{METHODS}

Search strategy: We searched the databases of Pubmed, Embase, the Cochrane Library, Web of Science for English language articles published from January 1999 to October 2019, involving all fertility-preserving treatments for young, grade 1 presumed stage IA EC. We combined Medical Subject Headings (MeSH) with keyword search. For each database, we retrieved 5 keywords: EC, fertility preservation, hysteroscopic resection, progesterone, levonorgestrelreleasing intrauterine system (LNG-IUS) and their related words and form a retrieval mode for retrieval.

Participant or population: Patients with early-stage endometrial cancer.

Intervention: a) taking oral progestin only therapy, b) hysteroscopic resection combined with progestin /LNG-IUS/GnRHa, c) Levonorgestrel-releasing intrauterine system (LNG-IUS) or combined with progestin/GnRH-a.

Comparator: a) taking oral progestin only therapy, b) hysteroscopic resection combined with progestin /LNG-IUS/GnRH$a, c)$ Levonorgestrel-releasing intrauterine system (LNG-IUS) or combined with progestin/GnRH-a.

Study designs to be included: To collect the research literature on fertility preservation therapy in patients with early-stage welldifferentiated EC aged $\leq 40$ years from January 1999 to October 2019. The inclusion was performed using $R$ software (version R3.5.3) meta-analysis of single rate. By comparing the complete remission rate (CRR), recurrence rate (ReR), pregnancy rate (PregR) and live birth rate (LBR).

Eligibility criteria: a) those women aged $\leq 40$ years who had a strong desire to preserve their reproductive function; b) histopathologically confirmed welldifferentiated endometrial adenocarcinoma; (c) no myometrium infiltration and involvement of cervical parenchyma detected and no paracasal metastasis detected by transvaginal ultrasonography (TVS) or magnetic resonance imaging (MRI); (d) positive progesterone receptors (PR); (e) no medication contraindications for progesterone.

Information sources: We searched the databases of Pubmed, Embase, the Cochrane Library, Web of Science for English language articles published from January 1999 to October 2019.

Main outcome(s): By comparing the complete remission rate (CRR), recurrence rate (ReR), pregnancy rate (PregR) and live birth rate (LBR), we indirectly evaluated the efficiency of the three fertility-preserving treatments: a) taking oral progestin only therapy, b) hysteroscopic resection combined with progestin /LNG-IUS/GnRHa, c) Levonorgestrel-releasing intrauterine system (LNG-IUS) or combined with progestin/GnRH-a.

Quality assessment / Risk of bias analysis: MINORS scale were used for quality evaluation.In this process, two researchers independently screened the literature, 
extracted and cross-checked the data. Any disagreement was resolved through discussion or judged by the third researcher.

Strategy of data synthesis: The extracted data include: a) basic information of the study included: title, author and contact information, year of publication, time of research, country; b) basic characteristics of the research subjects: research type, age of subjects and specific interventions; c) risk assessment of bias included in the study and quality assessment ; d) main data of associated outcome indicators: total number of samples, number of remission, number of recurrence, number of pregnancy, number of pregnancy preparation, number of births, pregnancy pattern, remission time, recurrence time, follow-up time; outcome measurement parameters: remission rate, ReR, PregR, fertility rate.

Subgroup analysis: Study: clinical case analysis ande cohort studies. The inclusion was performed using $\mathbf{R}$ software (version R3.5.3) meta-analysis of single rate.

Sensibility analysis: By changing the type of study in a particular study, the results were reanalyzed to see if there were any changes.

Language: English.

Country(ies) involved: China.

Keywords: Endometrial cancer; Fertility preservation; Hysteroscopic resection; Progestin; Levonorgestrel-releasing intrauterine system.

Contributions of each author:

Author 1 - Zhao Xiaoli.

Author 2 - Zhao Sufen. 